\title{
A CANARD MECHANISM FOR LOCALIZATION IN SYSTEMS OF GLOBALLY COUPLED OSCILLATORS*
}

\author{
HORACIO G. ROTSTEIN ${ }^{\dagger}$, NANCY KOPELL ${ }^{\dagger}$, ANATOL M. ZHABOTINSKY $^{\ddagger}$, AND \\ IRVING R. EPSTEIN $\ddagger$
}

\begin{abstract}
Localization in a discrete system of oscillators refers to the partition of the population into a subset that oscillates at high amplitudes and another that oscillates at much lower amplitudes. Motivated by experimental results on the Belousov-Zhabotinsky reaction, which oscillates in the relaxation regime, we study a mechanism of localization in a discrete system of relaxation oscillators globally coupled via inhibition. The mechanism is based on the canard phenomenon for a single relaxation oscillator: a rapid explosion in the amplitude of the limit cycle as a parameter governing the relative position of the nullclines is varied. Starting from a parameter regime in which each uncoupled oscillator has a large amplitude and no other periodic or other stable solutions, we show that the canard phenomenon can be induced by increasing a global negative feedback parameter $\gamma$, with the network then partitioned into low and high amplitude oscillators. For the case in which the oscillators are synchronous within each of the two such populations, we can assign a canard-inducing critical value of $\gamma$ separately to each of the two clusters; localization occurs when the value for the system is between the critical values of the two clusters. We show that the larger the cluster size, the smaller is the corresponding critical value of $\gamma$, implying that it is the smaller cluster that oscillates at large amplitude. The theory shows that the above results come from a kind of self-inhibition of each cluster induced by the local feedback. In the full system, there are also effects of interactions between the clusters, and we present simulations showing that these nonlocal interactions do not destroy the localization created by the self-inhibition.
\end{abstract}

Key words. canard phenomenon, globally coupled oscillators, relaxation oscillator, localization of oscillations

AMS subject classifications. $34 \mathrm{C} 15,34 \mathrm{C} 26$

DOI. $10.1137 / \mathrm{S} 0036139902411843$

1. Introduction. The Belousov-Zhabotinsky (BZ) reaction is the prototype system in nonlinear chemical dynamics $[1,2,3]$ (see references therein). In bulk, it is a relaxation oscillator. A wide variety of spatially extended patterns have been found in experiments on this reaction. Along with the experiments, chemically plausible mathematical models have been proposed and studied both analytically and numerically. The results obtained qualitatively reproduce experimental findings. Recently, new patterns have been found as nondiffusive couplings have been experimentally and numerically introduced.

In particular, the existence of localized oscillatory clusters has been reported in $[4,5]$ for the BZ reaction with global inhibitory feedback. Simulations performed on the Oregonator model [5] and a modified Oregonator model [6] of the BZ reaction, both with global inhibitor feedback, reproduce the experimental findings. However, the mechanism by which localized cluster formation occurs remains unclear from both the mathematical and chemical points of view.

* Received by the editors July 22, 2002; accepted for publication (in revised form) February 28, 2003; published electronically September 4, 2003.

http://www.siam.org/journals/siap/63-6/41184.html

${ }^{\dagger}$ Department of Mathematics and Center for Biodynamics, Boston University, Boston, MA 02215 (horacio@bu.edu, nk@bu.edu). The work of the first author was partially supported by the Burroughs Wellcome Fund. The work of the second author was partially supported by NSF grant DMS-9706694.

$¥$ Department of Chemistry and Volen Center for Complex Systems, Brandeis University, Waltham, MA 02454 (zhabotin@brandeis.edu, epstein@brandeis.edu). The work of the third and fourth authors was partially supported by NSF grant CHE-9988463. 
We consider as a cluster a set of "cells" or "chemical points" of the reactor that oscillate synchronously with the same amplitude. In certain cases, depending on some parameter, only two different amplitude regimes of oscillations occur: large amplitude oscillations (LAO) and small amplitude oscillations (SAO). The LAO regime consists of limit cycles whose amplitudes are $\mathrm{O}(1)$, i.e., almost equal to the maximum amplitude of the limit cycle for a single uncoupled oscillator, whereas the SAO regime consists of limit cycles whose amplitudes are of order of magnitude $\epsilon \ll 1$. In such cases there is a range of amplitudes that is not observable because they occur in an exponentially small interval of the governing parameter. When the system is divided into two or more clusters and at least one oscillates in an LAO regime and one in an SAO regime, we say that the clusters are localized. Clusters that are in the same amplitude regime may oscillate with a small difference in their amplitudes (compared with the LAO), but we do not refer to that situation as a localization phenomenon. Note that our definition of clusters does not require oscillators in each cluster to be spatially grouped; we disregard spatial structure in this work.

The localized cluster patterns found in the experiments and simulations on the BZ reaction with global inhibitory feedback $[4,5,6]$ present two main features that might seem counterintuitive:

1. Two different oscillatory regimes coexist in a system of identical coupled oscillators.

2. The cluster with the largest number of oscillators is always in the SAO regime whereas the smallest clusters are in an LAO regime; one might expect the largest cluster to be oscillating in an LAO regime and suppressing the smaller ones as occurs in other systems, e.g., neural systems with inhibitory synapses, with all-to-all identical coupling. In this latter case, if one cluster has a larger number of cells than the others, the former can suppress the latter.

In this paper we seek to explain the mechanism of localization for globally coupled relaxation oscillators of the FitzHugh-Nagumo (FHN) type, along with the two features mentioned above. The FHN-type models were chosen as simplifications of the Oregonator models. Although they are not precise as descriptions of chemical phenomena, they display the localization phenomenon and are easier to study analytically in order to give some insight into the dynamical mechanisms that produce localized solutions. In addition, they display some of the relevant qualitative features of the modified Oregonator model studied in [6], such as the shape of the nullclines, the fact that the limit cycle is created in a supercritical Hopf bifurcation, and the relaxation nature of the oscillator. In a forthcoming paper we will address the questions related to the mechanism of localization in the modified version of the Oregonator used in $[6,7]$.

We argue that the mechanism of localization is based on the canard phenomenon that occurs in single relaxation oscillators. The canard phenomenon is a very rapid change in the amplitude of the limit cycle of a relaxation oscillator as the inhibitor nullcline moves with respect to the activator nullcline $[11,12,13,14,15,16]$. It arises in the context of experiments and simulations of nonlinear chemical dynamics $[17,18,19]$ and in a two-pool model describing the mechanism of calcium-induced calcium release $[20,21]$.

The mechanism of localization in oscillatory systems, not of the relaxation type, has been studied in $[8,9,10]$ for nonidentical diffusively coupled oscillators. To our knowledge, the mechanism of localization in relaxation-type oscillators has not been analyzed before. 
In section 2 we present a general formulation for a class of models of globally coupled oscillators of FHN type that include the modified FHN (MFHN) models studied in this manuscript. For single FHN-type oscillators, the fast variable nullcline is cubic-like and intersects, on its middle branch, the slow variable nullcline, an increasing function. The parameters were chosen such that this intersection is an unstable fixed point. In section 3 we explain the reduction of dimensions strategy, a self-consistent argument that reduces the dimensionality of the mathematical problem by assuming the existence of $M$ clusters, each with a different dynamical behavior (different amplitudes, phases or both). Within each cluster, the oscillators synchronize.

In section 4 we describe the canard phenomenon for a single FHN-type equation and review some results. Following [15], we present a mathematical expression for an asymptotic approximation to the "canard critical value" for the parameter $\lambda$, the parameter responsible for the displacement of the slow variable nullcline relative to the fast variable nullcline, as a function of the remaining parameters of the model. When, by increasing or decreasing $\lambda$ through a critical value $\lambda_{c}$, there is a sudden change (of canard type) in the amplitude of the limit cycle, we say that the canard phenomenon has been induced by changes in $\lambda$, and we call $\lambda_{c}$ the canard critical value of $\lambda$. Strictly speaking, the sudden change in the amplitude of the limit cycle takes place in an exponentially small interval of values of $\lambda$; the canard critical value is the limit of the interval as $\epsilon \rightarrow 0$.

In section 5 we show that, when there are synchronized (bulk) oscillations for the globally coupled system (only one cluster), the canard phenomenon may be induced by increasing the value of the global feedback parameter $\gamma$ and keeping $\lambda$ fixed. In the FHN-type models with global feedback presented here, as well as in the BZ model with global feedback used in [6], the intersection point between nullclines remains fixed as $\gamma$ is increased. When $\gamma=0$ (no global feedback) the uncoupled oscillators are in an LAO regime; localization for these models is a consequence of the global coupling. An asymptotic approximation to the critical global feedback value, $\gamma_{c}$, is also calculated as a function of $\lambda$ and other parameters of the model. These results are the basis of our analysis of the localization phenomenon.

The localization phenomenon for a two-cluster system, in which one cluster is in an LAO regime and the other is in an SAO regime, is analyzed in section 6. The dynamics of the two-cluster globally coupled system is analyzed by studying each cluster separately and considering the other cluster as forcing it. Under specific assumptions, this dynamics is a combination of self-inhibition of each cluster, responsible for creating an interval of values of $\gamma$ within which a localized solution may exist, and inhibition (forcing) exerted on each cluster by the remaining ones. We show that self-inhibition is stronger the larger the cluster size, which explains why in a localized solution the largest cluster is in an SAO regime. We show that, for the special case of the van der Pol (VDP) equations with global feedback, localization is produced by only the self-inhibition, and the forcing exerted on each cluster by the other does not affect localization. In this paper we analyze only the effect of self-inhibition; however, we present some simulations of other globally coupled FHN systems that support our claim that the localization phenomenon is present with the same features predicted theoretically. In section 7 , we relate our results to experiments and simulations.

2. Models. In this paper we study models of the type

$$
\left\{\begin{array}{l}
v_{k}^{\prime}=F\left(v_{k}, w_{k}\right)-\gamma(\langle w\rangle-\bar{w}), \\
w_{k}^{\prime}=\epsilon G\left(v_{k}, w_{k} ; \lambda\right)
\end{array}\right.
$$




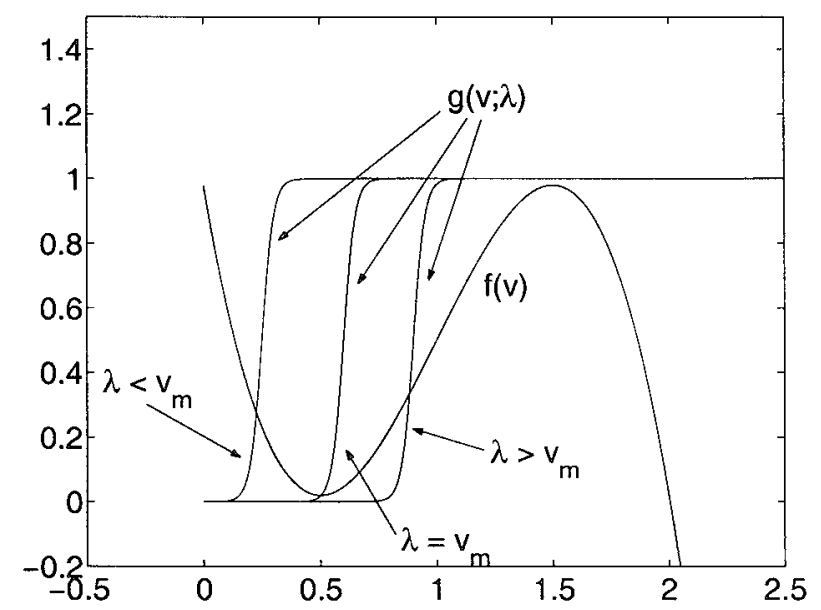

FIG. 1. Nullclines for a sigmoid version of the FHN model for several values of $\lambda=\bar{v}-v_{\text {min }}$ (the intersection point between nullclines).

for $k=1, \ldots, N$ and $0<\epsilon \ll 1$. In $(1), F(v, w)$ is such that the zero level curve $F(v, w)=0$ can be expressed as $w=f(v)$ with $f(v)$ a cubic-like function having one local minimum at $\left(v_{m}, w_{m}\right)$ and one local maximum at $\left(v_{M}, w_{M}\right)$ with $v_{m}<v_{M}$ and $w_{m}<w_{M}$. The function $G$ is a nonincreasing function of $w$ such that the zero level curve $G(v, w ; \lambda)=0$ is an increasing function of $v$ for every $\lambda$ in a given neighborhood of $\lambda=0$ and is also a decreasing function of $\lambda$ for all $v$ in a neighborhood of $v_{m}$. We further assume that $F=0$ and $G=0$ intersect at $(\bar{v}, \bar{w})$ with $\bar{v}=v_{m}$ when $\lambda=0$ and that $(\bar{v}, \bar{w})$ is an unstable fixed point lying on the central branch of $f$ when $\lambda>0$. The constant $\gamma$ is the global feedback parameter, and $\langle w\rangle$ is given by

$$
\langle w\rangle=\frac{1}{N} \sum_{k=1}^{N} w_{k} .
$$

Note that $(\bar{v}, \bar{w})$ does not depend on $\gamma$, as we can see by replacing $\langle w\rangle$ by $\bar{w}$ in (1). In all models considered here, the systems are assumed to be in a relaxation oscillatory regime in the absence of global coupling $(\gamma=0)$. For $\gamma=0$, changes in the parameter $\lambda$ alter the position of the $w_{k}$ nullcline (see Figure 1). When this nullcline moves, the intersection point $(\bar{v}, \bar{w})$ changes. As we will explain in section 4 , without loss of generality we can redefine $\lambda$ such that $\lambda=\bar{v}$. In the literature $v$ is usually referred to as the activator or the "potential" variable and $w$ as the "inhibitor" or the recovery variable.

Some specific systems may be modeled by making simplifying assumptions on (1) and considering $F(v, w)=f(v)-w$ and $G(v, w ; \lambda)=g(v ; \lambda)-w$, where $f$ is as described before and $g$ is an increasing function of $v$ for every $\lambda$ in a given neighborhood of $\lambda=0$ and a decreasing function of $\lambda$ for all $v$ in a neighborhood of $v_{m}$. Examples are

(i) VDP equations in Lienard form

$$
f(v)=-v^{3}+v^{2}, \quad G(v, w ; \lambda)=v-\lambda ;
$$

(ii) the classical FHN equations

$$
f(v)=-h v^{3}+a v^{2}-b v+c, \quad g(v ; \lambda)=\beta v-\eta,
$$

where $h, a, b, c, \beta$, and $\eta$ are nonnegative constants; 


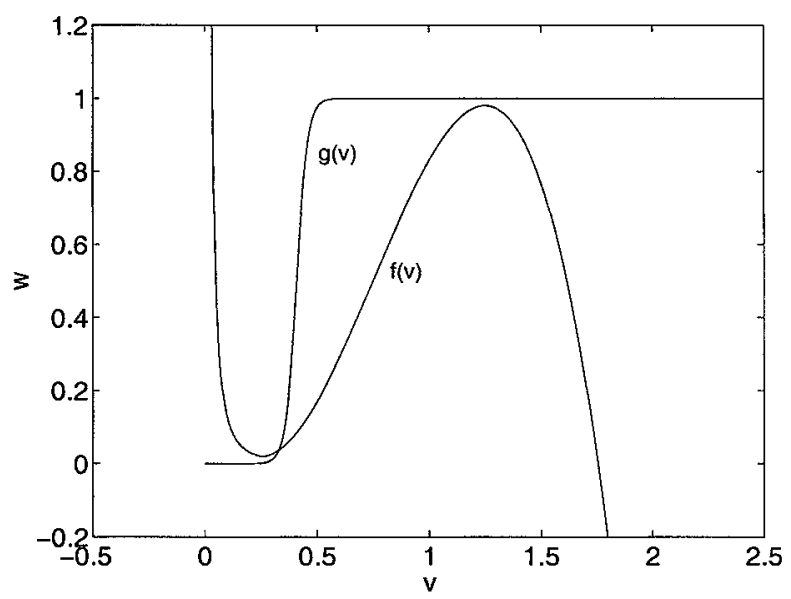

FIG. 2. Nullclines for the MFHN model for a single oscillator (or equivalently for $\gamma=0$ ). The values of the parameters used in our simulations are $h=1.92, a=4.32, b=1.8, c=0.23$, $\beta=0.41, \eta=0.05, \epsilon=0.05, v_{m}=0.25, f\left(v_{m}\right)=0.02, \bar{v}=0.328, \bar{w}=0.036092$. The function $f$ was constructed in such a way that the maximum and minimum are close to 1 and 0, respectively, in the following way: (i) we took a cubic function with minimum 0 at $v=0$ and maximum 0.98 at $v=1$, (ii) we shifted it up by 0.02 , (iii) we shifted it to the right by 0.25 . The function $g$, a sigmoid function, was built in such a way that it crosses $f$ at a single point, $\bar{v}$, placed to the right of the Hopf bifurcation and such that $\bar{v}>\lambda_{c}$ (beyond the canard critical value for a single oscillator). Note that $g$ is very steep and $\lim _{v \rightarrow \pm \infty}= \pm 1$.

(iii) the sigmoid FHN equations

$$
f(v)=-h v^{3}+a v^{2}-b v+c, \quad g(v ; \lambda)=\frac{1}{2}(\tanh ((v-\beta) / \eta)+1),
$$

where $h, a, b, c, \beta$, and $\eta$ are nonnegative constants; and

(iv) the MFHN equations, which we use in our simulations,

$$
\begin{aligned}
& f(v)= \begin{cases}f_{\text {cub }}(v), \\
f_{\text {cub }}\left(v_{m}\right) v_{m}^{2} / v^{2}, & v \geq v_{m},\end{cases} \\
& g(v ; \lambda)=\frac{1}{2}(\tanh ((v-\beta) / \eta)+1),
\end{aligned}
$$

and

$$
f_{c u b}=-h v^{3}+a v^{2}-b v+c .
$$

Here $v_{m}$ is the minimum of $f_{c u b} ; a, b, c, h, \beta, \eta$, and $\epsilon$ are nonnegative constants. In our simulations we use the following values for the parameters: $h=1.92, a=4.32$, $b=1.8, c=0.23, \beta=0.41, \eta=0.05$, and $\epsilon=0.05$. With those parameters we get $\left(v_{m}, w_{m}\right)=(0.25,0.02)$ and $(\bar{v}, \bar{w})=(0.328,0.036092)$. We can see the graph of the corresponding nullclines in Figure 2. In (4), (5), and (6) the parameter $\lambda$ (which was defined as the $v$-coordinate of the intersection point between the two nullclines of the system) is implicitly defined by other parameters of the model.

The MFHN model is a simplification of the modified version of the Oregonator model used in [6]; it allows an easier qualitative dynamical understanding by reproducing important aspects of the BZ dynamics and keeping some of its features, including 
the "N" shape of the nullcline corresponding to the first equation in (1), its asymptotic approach to the $w$ axis, its qualitative behavior as a function of the global feedback parameter, and an inhibitor dynamics described by a sigmoid function rather than a line. The motivation for using the MFHN system instead of more classical versions of the FHN system is that, by changing the global feedback parameter $\gamma$, we can find small amplitude limit cycles with smaller amplitude in the $v$ direction than for the FHN equations. This is due to the fact that the activator nullcline is asymptotic to the $w$ axis.

3. Strategy: Reduction of dimension using clusters. We are interested in localized solutions to (1)-(2) for $\gamma \neq 0$, in which two different portions of the system display LAO and SAO, respectively. Toward this end we will analyze the existence, properties, and stability of solutions to models of type (1)-(2) with $M$ different oscillatory behaviors $(M \leq N)$. More specifically, we will look for solutions to (1)-(2) in which the system of $N$ oscillators is divided into $M$ different sets, each set containing a fraction $\alpha_{k}, k=1, \ldots, M$, of the $N$ oscillators with $\sum_{k=1}^{M} \alpha_{k}=1$, and such that all oscillators in a set synchronize and oscillate with the same amplitude.

Since all the oscillators in each set are equivalent, we can write

$$
\langle w\rangle=\sum_{j=1}^{M} \alpha_{j} w_{j} .
$$

Bulk oscillations correspond to $M=1$. Two-phase (phase-locked) oscillations correspond to $M=2$, as do localized oscillations in which a fraction of the system oscillates with large amplitude and the rest of the system oscillates with small amplitude. $M=3$ includes three-phase (phase-locked) oscillations and localized oscillations in which a fraction of the system displays two-phase (phase-locked) LAO and the rest of the system oscillates with small amplitude.

In order to consider the influence of the rest of the system on the $k$ th oscillator, we define

$$
S_{k}=\sum_{j=1, j \neq k}^{M} \alpha_{j} w_{j}
$$

for $k=1, \ldots, M$. Using (9) and (1), we obtain

$$
\left\{\begin{array}{l}
v_{k}^{\prime}=F\left(v_{k}, w_{k}\right)-\gamma \alpha_{k} w_{k}+\gamma \bar{w}-\gamma S_{k}, \\
w_{k}^{\prime}=\epsilon G\left(v_{k}, w_{k} ; \lambda\right)
\end{array}\right.
$$

for $k=1, \ldots, M$. Note that the last term in the first equation of (10) is the only one depending on $w_{j}, j=1, \ldots, M, j \neq k$. This term can be seen as a forcing exerted by the rest of the oscillators on the $k$ th one. For $M=1\left(S_{k}=0, \alpha_{1}=1\right),(10)$ is an unforced oscillator with global coupling; it describes bulk oscillations of the whole system. For $M>1$, the inhibitor nullsurfaces are not dependent on $\gamma$ or $S_{k}$, while the activator nullsurfaces, which are solutions of

$$
F\left(v_{k}, w_{k}\right)-\gamma \alpha_{k} w_{k}+\gamma \bar{w}-\gamma S_{k}=0
$$

for $k=1, \ldots, M$, vary depending on $S_{k}$ and $\gamma$.

When $F(v, w)=f(v)-w$ in (10) we have FHN-type equations. In this case the activator nullsurfaces are given by

$$
w_{k}=\frac{f\left(v_{k}\right)+\gamma \bar{w}}{1+\gamma \alpha_{k}}-\frac{\gamma}{1+\gamma \alpha_{k}} S_{k}
$$


for $k=1, \ldots, M$. For each $k$, the solutions $\left(v_{k}, w_{k}\right)$ of the FHN-type equations can be considered as living in a three-dimensional space $\left(v_{k}, w_{k}, S_{k}\right)$. The activator nullsurfaces vary in the $S_{k}$ direction. As the system evolves, $S_{k}$ changes in a periodic fashion. For each value of $S_{k}$ we can consider the projection of (12) onto the $\left(v_{k}, w_{k}\right)$ plane. This gives us the possibility of looking at the phase space of the FHN-type equations for each oscillator separately as if it were two-dimensional, with the activator nullcline moving up and down periodically according to $S_{k}$, i.e., according to the dynamics of the rest of the $M-1$ oscillators. The intersection point in the $\left(v_{k}, w_{k}\right)$ plane between the projections of the inhibitor and activator nullsurfaces becomes a periodic function of $t$ that moves as the $w_{k}$ nullcline moves. We call the $v_{k}$-coordinate of this time-dependent intersection point $\lambda_{k}=\lambda_{k}(t)$ for $k=1, \ldots, M$. Thus, for systems of the form $f(v, w)=f(v)-w$, we can decompose the whole system into $M$ forced subsystems of FHN type, one for each value of $k$. The forcing exerted on one oscillator depends on the remaining ones.

Stability of a solution to (10) does not automatically imply stability with respect to the full equations (1), since the solution may not be stable to perturbations that destroy the clustering into groups of equivalent oscillators. Hence once a solution has been numerically found for a specific model and value of $M$, it is desirable to check its stability in the $N$-array of globally coupled oscillators. We approach this problem numerically. In order to numerically solve system (1) we used the modified Euler method [22] for $N=100$ with a step size $\Delta t=0.01$. For $M=2$ we divided the $N$ oscillators into two sets, each with uniform initial conditions. Once each set of oscillators (with $\alpha_{k} N$ oscillators belonging to each set, $k=1,2$ ) settled down in a specific limit cycle, we applied a random perturbation of maximum amplitude 0.001 to each variable. We applied the following criterion for stability: if, after the perturbation, each oscillator returns to its original limit cycle and phase difference, then we say that the system is $N$-stable (numerically stable in an array of $N$ oscillators). Otherwise we say that the system is $N$-unstable (numerically unstable in an array of $N$ oscillators). We are aware that our definition of stability is not a rigorous one and can be affected by numerical instabilities. Still, it gives us valuable information about the stability of phase and localized clusters for the subset of values of $\gamma$ for which they exist.

4. Canard phenomenon. In this section we review the canard phenomenon for relaxation oscillators. Consider system (1) for a single oscillator and $\gamma=0$; i.e.,

$$
\left\{\begin{array}{l}
v^{\prime}=F(v, w) \\
w^{\prime}=\epsilon G(v, w ; \lambda)
\end{array}\right.
$$

where $0<\epsilon \ll 1$ and where $F$ and $G$ are as described in section 2. We first look at FHN-type models; i.e., $F(v, w)=f(v)-w$ with $f$ as described in section 2 . We assume that system (13) is in an oscillatory regime. The nullclines for a sigmoid-type FHN model and a limit cycle corresponding to a chosen set of parameters are shown in Figure 3.

The dynamics of system (13) depends on the value of $\lambda$, i.e., on the relative position of the $w$ nullcline with respect to the $v$ nullcline. For the FHN-type equations there exists a Hopf bifurcation point $\lambda_{H}(\epsilon) \geq v_{m}$ in a neighborhood of $\left(v_{m}, w_{m}\right)$ which converges to $\left(v_{m}, w_{m}\right)$ as $(\epsilon, \lambda) \rightarrow(0,0)$ (see Appendix $\mathrm{C}$ and Figure 1). For values of $\lambda<v_{H}$ system (13) has a steady state as the only attractor, and the system is excitable $[1,3,23,24]$; i.e., relatively small perturbations (but large enough to exceed a threshold, a curve in phase space, determined by the $v$ nullcline and the parameters of the model) give rise to a large excursion that returns to the attractor. This trajectory 
a)

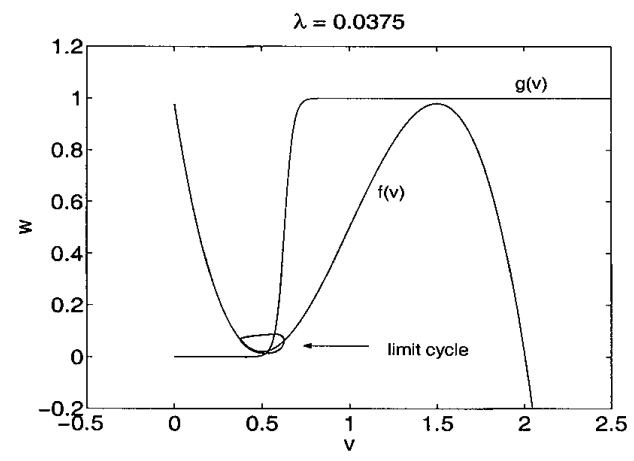

c)

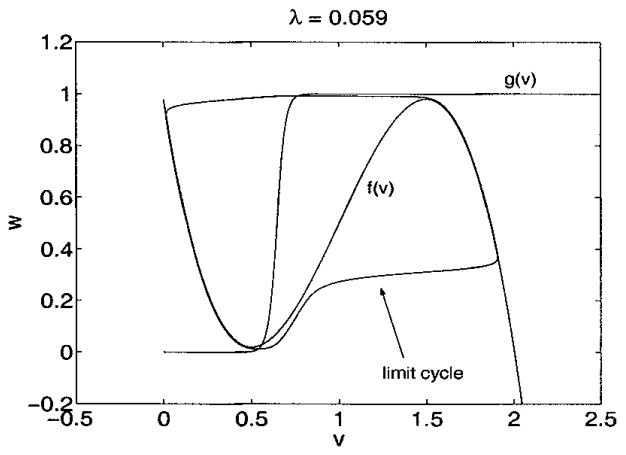

b)

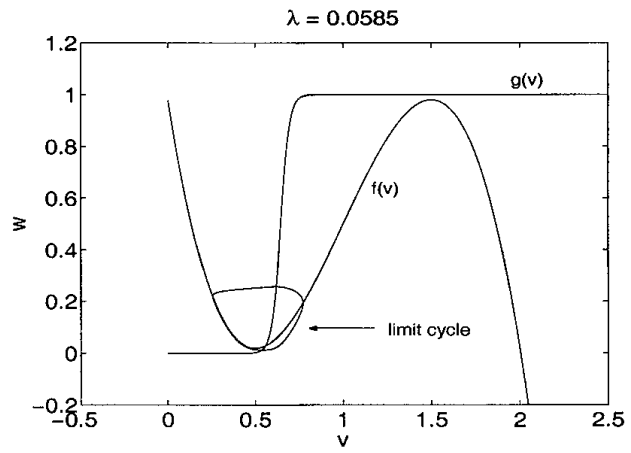

d)

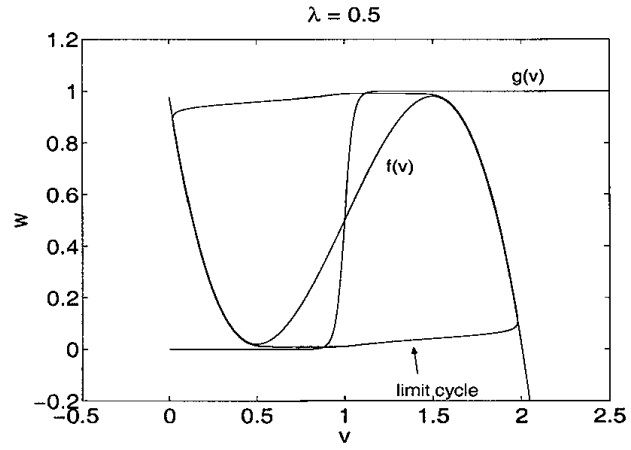

FIG. 3. Nullclines and limit cycle for a sigmoid version of the FHN model where $\lambda=\bar{v}-v_{\text {min }}$. The values of the parameters are as in Figure 2 and (a) $\lambda=0.0375$, (b) $\lambda=0.0585,(\mathrm{c}) \lambda=0.059$, (d) $\lambda=0.5$.

is usually called an excitation loop, a pulse, or a spike. Subthreshold perturbations return to the stable fixed point with no large excursion. At $\lambda=\lambda_{H}(\epsilon)$, system (13) undergoes a supercritical Hopf bifurcation. As $\lambda$ increases, the amplitude of the limit cycle increases slowly for small enough values of $\lambda$, part of the trajectory being very close to the unstable branch of the $v$ nullcline for a while, then crossing the unstable branch and moving toward the left branch of the $v$ nullcline, as illustrated in Figure $3(\mathrm{a})$ and 3(b). At some critical point $\lambda_{c}(\epsilon)>\lambda_{H}(\epsilon)$, the trajectory moves toward the right branch of the activator nullcline instead of moving toward the left branch, and the limit cycle expands rapidly (over an exponentially small interval in the parameter $\lambda$ ) becoming a relaxation oscillator $[1,3,25,26]$, as seen in the transition from Figure 3(b) to 3(c). After that, the amplitude of the limit cycle either increases slowly or remains constant as $\lambda$ is increased, until the oscillator becomes like the one in Figure $3(\mathrm{~d})$. By symmetry, when $\lambda$ is near the maximum of $w=f(v)$, the same effect is seen in a small neighborhood of the Hopf bifurcation near $\lambda=v_{M}$. In Figure 4 we can see the amplitude of the limit cycle, given by the maximum and minimum values of $v$ and $w\left(v_{\min }, v_{\max }, w_{\min }\right.$, and $\left.w_{\max }\right)$, as a function of $\lambda$ for the sigmoid version of the FHN equations (5). This rapid change from a "small" amplitude limit cycle to a "large" amplitude limit cycle is known as the canard phenomenon [11, 12, 14, 15, 16, 27]. In this case the canard phenomenon has been induced by changes in $\lambda$. Here we concentrate on the canard phenomenon near $v=v_{m}$. 
a)

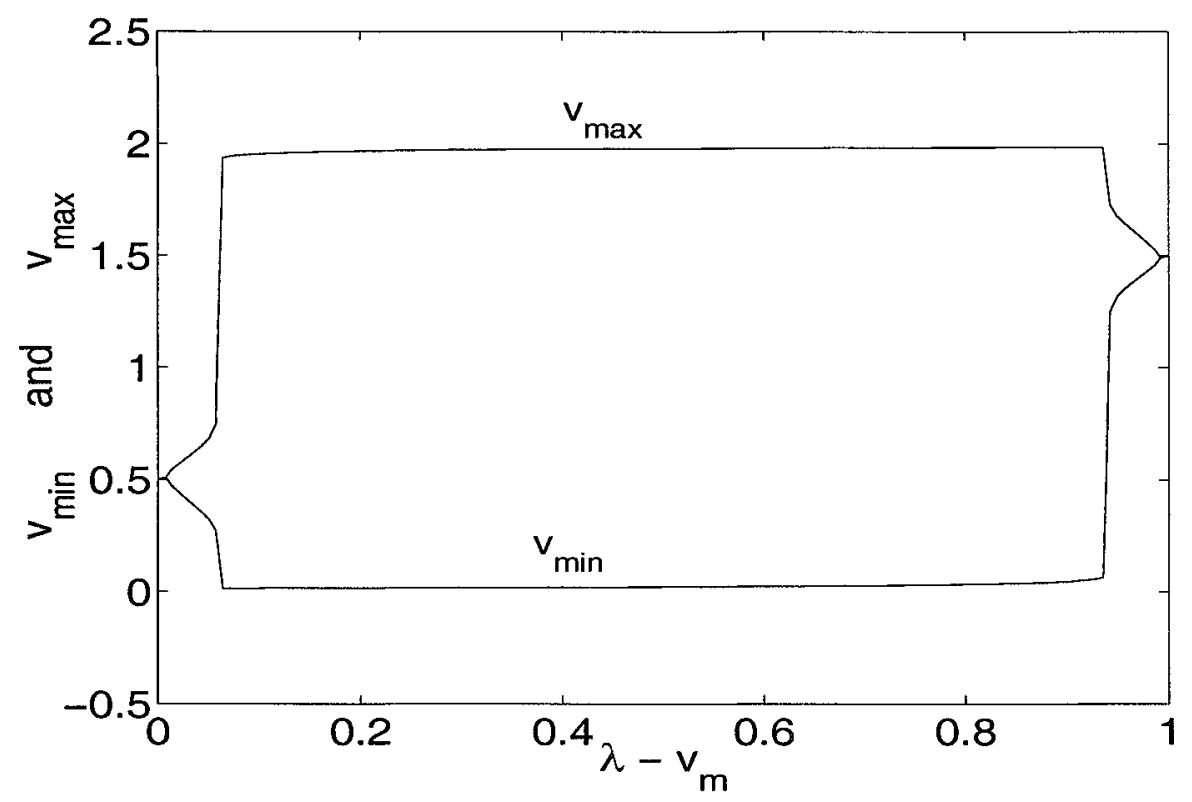

b)

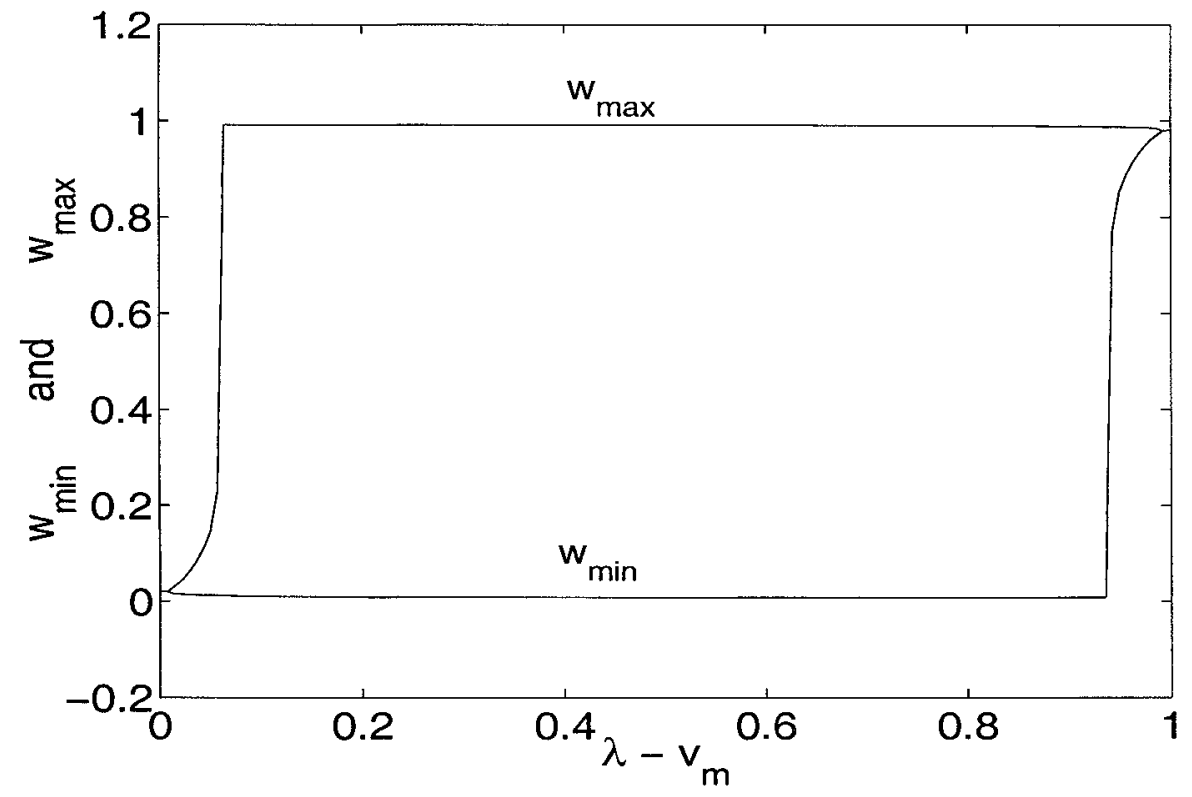

FIG. 4. Amplitude of the limit cycle as a function of the crossing point between the activator and inhibitor nullclines $\lambda$ for a single cell FHN oscillator. The values of the parameters are as in Figure 2 . (a) v-amplitude ( $v_{\min }$ and $\left.v_{\max }\right),(\mathrm{b})$-amplitude $\left(w_{\min }\right.$ and $\left.w_{\max }\right)$. 
The canard phenomenon was discovered by Benoit et al. [14] for the VDP oscillator. In their work [14] they show that there exists a critical value $\lambda_{c}(\epsilon)$ of $\lambda$ such that for $\lambda$ in a small neighborhood of $\lambda_{c}$ the limit cycle deforms into a curve similar to the one shown in Figure 3(c). While Benoit et al. [14] used nonstandard analysis techniques in their study, Eckhaus [12] and Baer and Emeux [13] used asymptotic techniques. In particular they found expressions for the canard critical value $\lambda_{c}$ for VDP-type equations and for a generalization of system (13).

The canard phenomenon for (13) has been also studied by Dumortier and Roussarie [11] and by Krupa and Szmolyan [15, 16]. We follow the latter authors in our approach. In order to present their results, without loss of generality, we take $\left(v_{m}, w_{m}\right)=(0,0)$. For $(v, w)=\mathbf{0}$ we assume that $F(\mathbf{0})=\mathbf{0}, \partial F / \partial v(\mathbf{0})=\mathbf{0}$, $\partial^{2} F / \partial v^{2}(\mathbf{0}) \neq \mathbf{0}$, i.e., $\left(v_{m}, w_{m}\right)$ is a nondegenerate local minimum (fold point) of the nullcline $F(v, w)=0$ for $\lambda$ in a suitable interval. Furthermore, $\partial F / \partial w(\mathbf{0}) \neq \mathbf{0}$. We also assume for $(v, w, \lambda)=\mathbf{0}$ that $G(\mathbf{0})=\mathbf{0}, \partial G / \partial v(\mathbf{0}) \neq \mathbf{0}$, and $\partial G / \partial \lambda(\mathbf{0}) \neq \mathbf{0}$. These conditions defining a canard point (which will be referred to as canard conditions) mean that the nullcline $G(v, w, \lambda)=0$ is transverse to the nullcline $F(v, w)=0$, and it passes through the fold point with nonzero speed as $\lambda$ varies. As pointed out above, $\bar{v}$ increases as $\lambda$ increases (see Figure 1), allowing us to reparametrize $\lambda$ such that $\lambda=\bar{v}$. For the VDP and FHN equations, these assumptions are satisfied with an appropriate change of variables.

We show in Appendix B that

$$
\lambda_{c}=\Lambda \epsilon+\left|F_{w}\right| \Upsilon \epsilon+\mathcal{O}\left(\epsilon^{3 / 2}\right)
$$

where

$$
\Upsilon=\frac{G_{v}}{2 F_{v v}\left|G_{\lambda}\right|}\left(\frac{G_{v}}{F_{v v}}\right)_{v}, \quad \Lambda=-\frac{G_{v}}{2 F_{v v}^{3}\left|G_{\lambda}\right|}\left(G_{v} F_{v w} F_{v v}+G_{w} F_{v v}^{2}\right),
$$

and all the functions are calculated at $\mathbf{0}$. To obtain (14) we used an earlier result by Krupa and Szmolyan [15]. There, the cubic-like function was assumed to have its minimum at $(0,0)$.

For the VDP equations (3), $\Lambda=0$ and $\Upsilon=-f^{\prime \prime \prime}(0) / 2\left(f^{\prime \prime}(0)\right)^{2}=3 / 4$, coinciding with the expression found by Eckhaus [12]. For the classical FHN equations (4), $\Lambda=\beta /\left(2 f^{\prime \prime}(0)\left|g_{\lambda}\right|\right)$ and $\Upsilon=-\beta f^{\prime \prime \prime}(0) /\left(2\left(f^{\prime \prime}(0)\right)^{3}\left|g_{\lambda}\right|\right)$. The expression for the canard critical value becomes

$\lambda_{c}=\frac{\beta}{2\left(f^{\prime \prime}(0)\right)^{3}\left|g_{\lambda}(0)\right|}\left[\left(f^{\prime \prime}(0)\right)^{2}-\beta f^{\prime \prime \prime}(0)\right] \epsilon+\mathcal{O}\left(\epsilon^{3 / 2}\right)=\frac{\beta\left(2 a^{2}+3 \beta h\right)}{8 a^{3}\left|g_{\lambda}(0)\right|} \epsilon+\mathcal{O}\left(\epsilon^{3 / 2}\right)$.

For the general FHN-type equations with $G(v, w ; \lambda)=g(v ; \lambda)-w$,

$$
\begin{gathered}
\lambda_{c}=\frac{g^{\prime}(0)}{2\left(f^{\prime \prime}(0)\right)^{3}\left|g_{\lambda}(0)\right|}\left[\left(f^{\prime \prime}(0)\right)^{2}-f^{\prime \prime \prime}(0) g^{\prime}(0)+g^{\prime \prime}(0) f^{\prime \prime}(0)\right] \epsilon+\mathcal{O}\left(\epsilon^{3 / 2}\right) \\
=\frac{g^{\prime}(0)}{8 a^{3}\left|g_{\lambda}(0)\right|}\left[2 a^{2}+3 h g^{\prime}(0)+a g^{\prime \prime}(0)\right] \epsilon+\mathcal{O}\left(\epsilon^{3 / 2}\right) .
\end{gathered}
$$

Note that if the minimum of the activator nullcline $\left(v_{m}, f\left(v_{m}\right)\right) \neq(0,0)$, a translation of coordinates may be performed without changing the values of the derivatives of $f$ and $g$. 
By construction, $f$ in the MFHN model is a matching of two different functions. The result is continuous but not differentiable at the origin. In order for the theory described in this section to be applicable, $F$ and $G$ must be $C^{k}$-functions with $k \geq 3$ (continuous at least up to the third derivative) [16]. In the analysis presented here we consider functions $f$ qualitatively similar to the MFHN function defined above, i.e., satisfying the canard conditions, but $C^{k}$ with $k \geq 3$. Our numerical simulations with the MFHN function qualitatively agree with the analytical predictions.

5. Canard phenomenon induced by the global feedback parameter in synchronized (bulk) oscillatory systems. In this section we study the influence of the global feedback parameter $\gamma$ on the amplitude regime (LAO or SAO) of the solution for $M=1$ (bulk or synchronized oscillations). In what follows, all functions are calculated at $\mathbf{0}$. For $M=1$, system (1) reads as

$$
\left\{\begin{array}{l}
v^{\prime}=F(v, w)-\gamma w+\gamma \bar{w} \\
w^{\prime}=\epsilon G(v, w ; \lambda)
\end{array}\right.
$$

We assume that at $\gamma=0, \lambda_{c}=\mathcal{O}(\epsilon)$ such that $\lambda_{c} \neq \mathcal{O}\left(\epsilon^{\nu}\right), \nu>1, \lambda=\mathcal{O}(\epsilon)$ fixed, and $\lambda>\lambda_{c}$; i.e., the system is in an LAO regime for $\gamma=0$.

First we explain how to apply the theory developed in section 4 to system (18). In the calculation of the canard critical value we use the fact that in a neighborhood of $\left(v_{m}, f\left(v_{m}\right)\right)=(0,0)$ the $v$ nullcline can be described by a parabolic function (see (48) in Appendix A). Then, by our assumption $\lambda=\mathcal{O}(\epsilon)(\bar{v}=\mathcal{O}(\epsilon))$, it follows that $\gamma \bar{w}=$ $\mathcal{O}\left(\epsilon^{2}\right)$. We can rescale the last term in the first equation in (18) by defining $\bar{w}=\kappa \epsilon^{2}$, getting the following expression for the $v$ nullcline: $\Phi(v, w, \epsilon):=F(v, w)-\gamma w+\gamma \kappa \epsilon^{2}$. Note that $\kappa$ is independent of $v$ and that $\Phi(0,0,0)=0$ as required in [15].

Remark. When the activator nullcline $\Phi=0$ is $\epsilon$-dependent, the canonical equations (48) are augmented by a term $\epsilon h_{6}(v, w, \epsilon)$ and the expression for the canard critical value has an extra term proportional to $h_{6, v}[15]{ }^{1}$ For $(18) h_{6}=\gamma \epsilon \kappa$, so $h_{6, v}=0$ and the expression for the canard critical value is not affected. The only effect of $\gamma$ on the canard critical value comes from the term $-\gamma w$ in the first equation in (18).

An expression for the canard critical value as a function of the global feedback parameter $\gamma$ can be calculated as in the calculation for $\gamma=0$ (see Appendix B) to obtain

$$
\lambda_{c}(\gamma)=\Lambda \epsilon+\left(\left|F_{w}\right|+\gamma\right) \Upsilon \epsilon+\mathcal{O}\left(\epsilon^{3 / 2}\right)=\lambda_{c}(0)+\gamma \Upsilon \epsilon+\mathcal{O}\left(\epsilon^{3 / 2}\right) .
$$

Expressions (19) and (15) imply that, by increasing the value of the global feedback parameter, the value of the canard critical value is increased, provided

$$
\left(\frac{G_{v}}{F_{v v}}\right)_{v}>0
$$

since $G_{v}$ and $F_{v v}$ were assumed to be positive. So, if for $\gamma=0$ we have $\lambda>\lambda_{c}(0)$ (the system is in an LAO regime), then the canard phenomenon can be induced by increasing the value of $\gamma$ without changing the value of $\lambda$. The change from LAO to SAO takes place in an interval of values of $\gamma$ exponentially small in $\epsilon$.

We now compute $\gamma_{c}$, the amount that $\gamma$ must be increased (in the limit as $\epsilon \rightarrow 0$ ) to induce the canard phenomenon, assuming that the system is in an LAO regime

\footnotetext{
${ }^{1}$ See the explanation after (53).
} 
when $\gamma=0$. Taking into account the assumptions made at the beginning of this section on $\lambda$ and $\lambda_{c}$, the critical value $\gamma_{c}(\lambda)$ of $\gamma$ may be calculated as the value of $\gamma$ that brings $\lambda_{c}(\gamma)$ to $\lambda$, i.e., by replacing $\gamma$ by $\gamma_{c}$ and $\lambda_{c}$ by $\lambda$, respectively, in (19):

$$
\lambda=\lambda_{c}(0)+\gamma_{c}(\lambda) \Upsilon \epsilon+\mathcal{O}\left(\epsilon^{3 / 2}\right) .
$$

From (14)

$$
\left|F_{w}\right| \Upsilon \epsilon=\lambda_{c}(0)-\Lambda \epsilon+\mathcal{O}\left(\epsilon^{3 / 2}\right)
$$

Substituting (22) into (21), multiplied by $\left|F_{w}\right|$, we get

$$
\left|F_{w}\right|\left[\lambda-\lambda_{c}(0)\right]=\gamma_{c}\left[\lambda_{c}(0)-\Lambda \epsilon\right]+\mathcal{O}\left(\epsilon^{3 / 2}\right) .
$$

Note that in the FHN models $\left|F_{w}\right|=\mathcal{O}(1)$, which has been used in the error term in (23). Rearranging terms and using (14), we get

$$
\text { (24) } \gamma_{c}(\lambda)=\left|F_{w}\right| \frac{\lambda-\lambda_{c}(0)}{\lambda_{c}(0)-\Lambda \epsilon}+\mathcal{O}\left(\frac{\epsilon^{3 / 2}}{\lambda_{c}(0)-\Lambda \epsilon}\right)=\left|F_{w}\right| \frac{\lambda-\lambda_{c}(0)}{\lambda_{c}(0)-\Lambda \epsilon}+\mathcal{O}\left(\epsilon^{1 / 2}\right) .
$$

For the VDP equations (3), $F_{w}=-1$ and $G$ is independent of $w$ so we have $\Lambda=0$. Thus from (19)

$$
\lambda_{c}(\gamma)=\lambda_{c}(0)(1+\gamma)+\mathcal{O}\left(\epsilon^{3 / 2}\right)
$$

and

$$
\gamma_{c}(\lambda)=\frac{\lambda-\lambda_{c}(0)}{\lambda_{c}(0)}+\mathcal{O}\left(\epsilon^{1 / 2}\right)
$$

Note that, since $G$ is independent of $w$, the nullclines intersect at the same value $\lambda$ for all $\gamma \geq 0$.

Using (19) and (24), the expressions for $\lambda_{c}$ and $\gamma_{c}$ for the FHN-type equations with $G(v, w ; \lambda)=g(v ; \lambda)-w$ are given by

$$
\lambda_{c}(\gamma)=\lambda_{c}(0)+\frac{\gamma g^{\prime}(0)}{2 f^{\prime \prime}(0)|g(\lambda)|}\left[\frac{g^{\prime \prime}(0) f^{\prime \prime}(0)-g^{\prime}(0) f^{\prime \prime \prime}(0)}{\left[f^{\prime \prime}(0)\right]^{2}}\right] \epsilon+\mathcal{O}\left(\epsilon^{3 / 2}\right)
$$

and

$$
\gamma_{c}(\lambda)=\frac{\lambda-\lambda_{c}(0)}{\lambda_{c}(0)}\left[1+\frac{g^{\prime}(0)}{2 \lambda_{c}(0) f^{\prime \prime}(0)\left|g_{\lambda}\right|} \epsilon\right]+\mathcal{O}\left(\epsilon^{1 / 2}\right) .
$$

In both cases, as $\gamma$ increases, the canard critical value moves to the right; then there exists a critical value of the global feedback parameter, $\gamma_{c}$, such that for values of $\gamma$ below (above) $\gamma_{c}$, solutions display LAO (SAO).

As noted above, for the MFHN model used in our simulations, we do not have an expression for the canard critical value as a function of the parameters of the model and $\gamma$, but we conjecture on the basis of numerical simulations that the behavior is similar to the smooth case described before. The results of numerical simulations are shown in Figures 5 and 6 . In Figure 5 we see the dependence of the amplitude of the limit cycle (represented by the minimum and maximum values of $v$ and $w$ ) on $\gamma$ for the MFHN model. We observe that for $\gamma=\gamma_{c}$ (in this case $\gamma_{c} \sim$ $0.429)$, there is a sudden change in both the $v$-and $w$-amplitudes of the limit cycle. 
a)

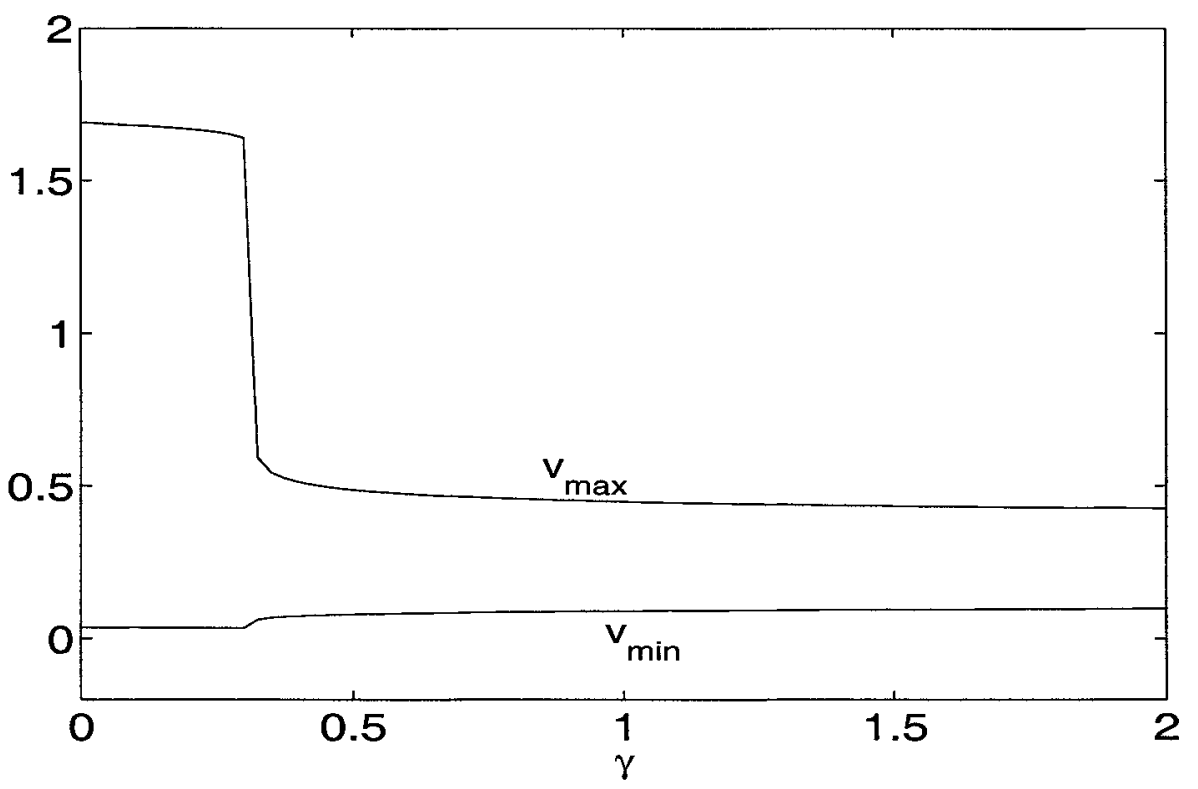

b)

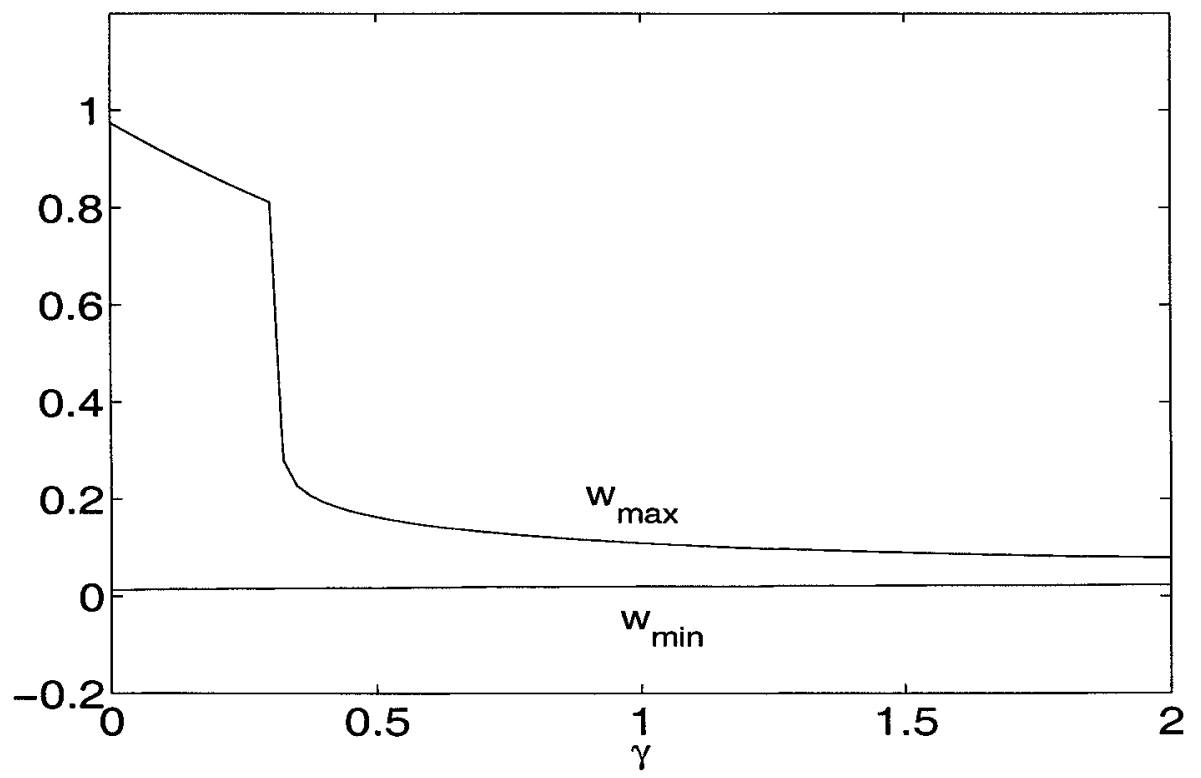

FIG. 5. Amplitude of the limit cycle for the MFHN oscillator as a function of the global feedback parameter $\gamma$. The values of the parameters are as in Figure 2. 
a)

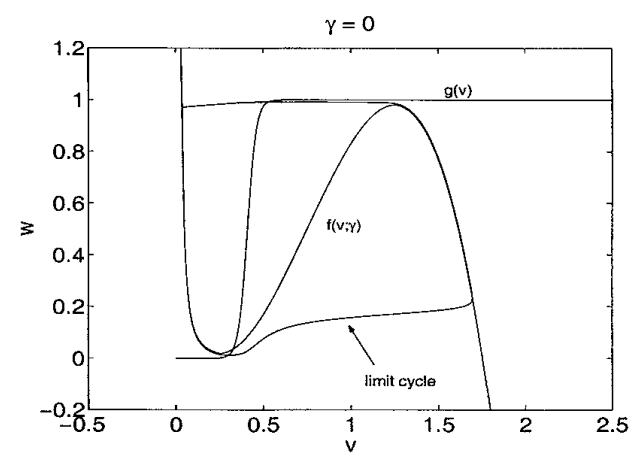

c)

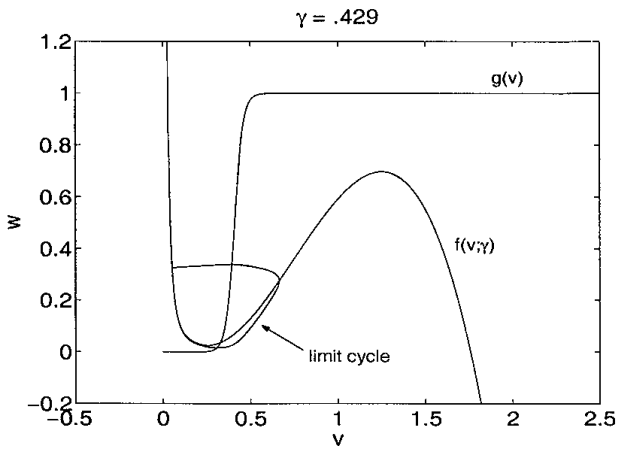

b)

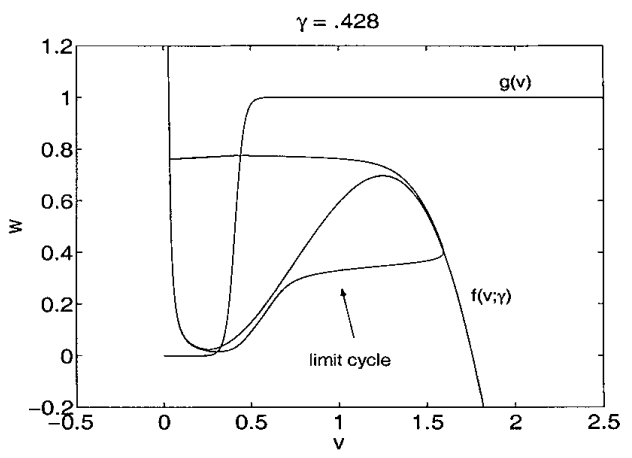

d)

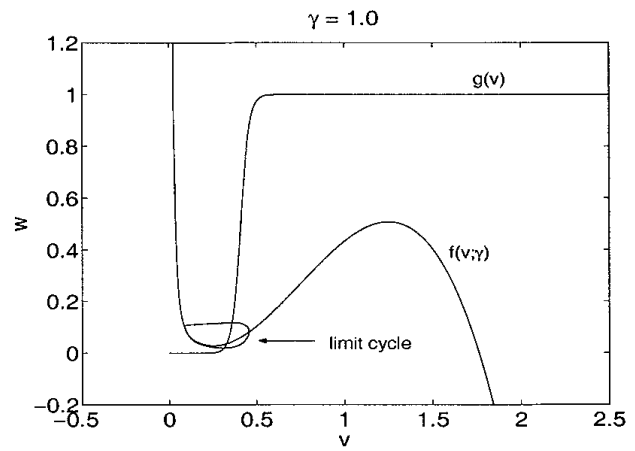

FIG. 6. Nullclines and phase plane for the MFHN oscillator for various values of $\gamma$. The function $f(v ; \gamma)=(f(v)+\gamma \bar{w}) /(1+\gamma)$. The values of the parameters are as in Figure 2 and (a) $\gamma=0$, (b) $\gamma=0.428$, (c) $\gamma=0.429$, (d) $\gamma=1$.

In Figure 6 we show the shape of the limit cycle for several values of $\gamma$ above and below $\gamma_{c}$. For $\gamma=0$ (Figure 6(a)) the system is in a relaxation oscillation regime. As $\gamma$ increases, the limit cycle goes through the lower knee of the activator nullcline, comes up along the unstable branch for a while, and then moves rapidly to the right branch of the activator nullcline (Figure 6(b)) if $\gamma<\gamma_{c}$; if $\gamma>\gamma_{c}$, the trajectory crosses the unstable branch and moves rapidly to the left branch of the activator nullcline (Figure 6(c)).

Our numerical simulations show that bulk oscillations for the MFHN model are 100-stable for $\gamma \leq 0.39$ and $\gamma \geq 25.0$.

6. Localized solutions. In this section we analyze the existence of localized solutions for a system of globally coupled FHN-type equations, i.e., equations (1)-(2), where $F(v, w)=f(v)-w$. We deal here with the case $M=2$; this can be easily generalized to larger values of $M$. In a two-cluster localized solution, some of the oscillators are in an SAO regime while the other oscillators are in an LAO regime.

By applying the reduction of dimensions described in section 3 we reduce the system of $N$ oscillators to a system of two oscillators. The activator nullcline for each oscillator is given by (12) for $k=1,2$. The first term in (12) depends only on $v_{k}$ and the second term is independent of $\left(v_{k}, w_{k}\right)$ and is the only one depending on $w_{j}$, $j=1,2, j \neq k$. As explained in section 3 , we can consider the second term in (12) 
as moving the nullcline, whose shape is given by the first term in (12), up and down. We call $\lambda_{k, c}(\gamma)$ and $\gamma_{k, c}(\lambda)$ the canard critical value and the critical global feedback parameter value, respectively, for $k=1,2$. Looking at each of the two oscillators separately we can calculate the respective canard critical values as a function of $\gamma$ and the fraction of oscillators in each cluster, $\alpha_{k}$, following the same reasoning leading to (19) and (24) in section 5 , where $\gamma$ is replaced by $\alpha_{k} \gamma$. This yields

$$
\lambda_{k, c}(\gamma)=\lambda_{c}(0)+\alpha_{k} \gamma \Upsilon \epsilon+\mathcal{O}\left(\epsilon^{3 / 2}\right)
$$

and

$$
\gamma_{k, c}(\lambda)=\frac{1}{\alpha_{k}} \frac{\lambda-\lambda_{c}(0)}{\lambda_{c}(0)-\Lambda \epsilon}+\mathcal{O}\left(\epsilon^{1 / 2}\right)
$$

for $k=1,2$. Thus, for a given $\gamma$, the larger $\alpha_{k}$ the larger the canard critical point for the $k$ th oscillator and the smaller the corresponding $\gamma_{k, c}$, i.e., the less the global feedback needed to get SAO. We can easily calculate

$$
\lambda_{1, c}(\gamma)-\lambda_{2, c}(\gamma)=\left(\alpha_{1}-\alpha_{2}\right) \gamma \Upsilon \epsilon+\mathcal{O}\left(\epsilon^{3 / 2}\right)
$$

and

$$
\gamma_{2, c}-\gamma_{1, c}=\frac{\alpha_{1}-\alpha_{2}}{\alpha_{1} \alpha_{2}} \frac{\lambda-\lambda(0)}{\lambda_{c}(0)-\Lambda \epsilon}+\mathcal{O}\left(\epsilon^{1 / 2}\right)
$$

For the VDP equations, the value of $\lambda_{k}(t)$ (see section 3 for the definition of this quantity) does not depend either on $k$ or on $t$. Let us refer to it as $\lambda$. In this case, expression (31) implies that if $\alpha_{1} \neq \alpha_{2}$, then we can find values of the global feedback parameter $\gamma$ for which $\lambda$ has a value between $\lambda_{1, c}$ and $\lambda_{2, c}$, thus producing a localized solution. As we can see from (31) and (32) the interval of values of $\lambda$ and $\gamma$ for which we can expect a localized solution increases with the difference between the fractions of oscillators in the two clusters. Since $\lambda_{k}(t)$ is independent of $k$ and $t$, localization in the VDP model is a consequence only of nonsymmetric self-inhibition, i.e., not a consequence of the forcing that the oscillators exert on one another. Note that the cluster with the larger $\alpha_{k}$ is the one in the SAO regime, as seen in experiments and simulations on the BZ reaction with global feedback. (In the latter case the LAO regime consisted of two phase locked clusters.) The shape, frequency, and amplitude of each limit cycle (considered separately) in the localized solutions depend, in ways that are not yet fully understood, on $\gamma$, on the size of the other oscillator, and possibly on other quantities.

For the FHN-type equations self-inhibition creates intervals of critical values of $\lambda$ and $\gamma$ given by (31) and (32), respectively. In contrast to the VDP equations, when $\gamma>0, \lambda_{k}(t)$ (see section 3 for the definition of this quantity) depends on both $k$ and $t$. The forcing exerted on each oscillator by the other one changes the value of $\lambda_{k}(t)$. Thus there are two effects we must consider in understanding how localized solutions arise: self-inhibition and external inhibition or forcing. How external forcing interacts with localization is not yet understood. However, our simulations for the MFHN model show that localization is present as expected and that the numerically determined interval $\gamma_{2, c}-\gamma_{1, c}$ in which there is localization increases as $\alpha_{1}-\alpha_{2}$ increases. In Figure 7 we show the amplitude of the solutions to the MFHN system with $M=2$ as a function of the global feedback parameter $\gamma$ for different values 
a)

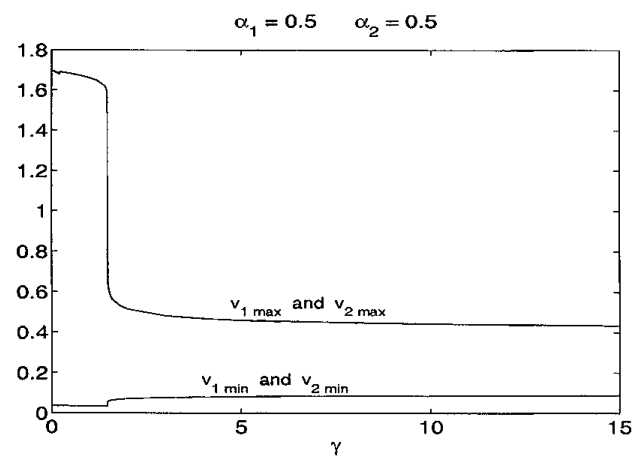

c)

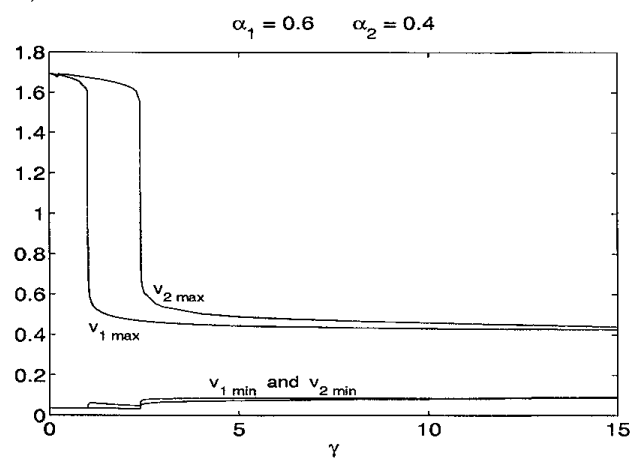

e)

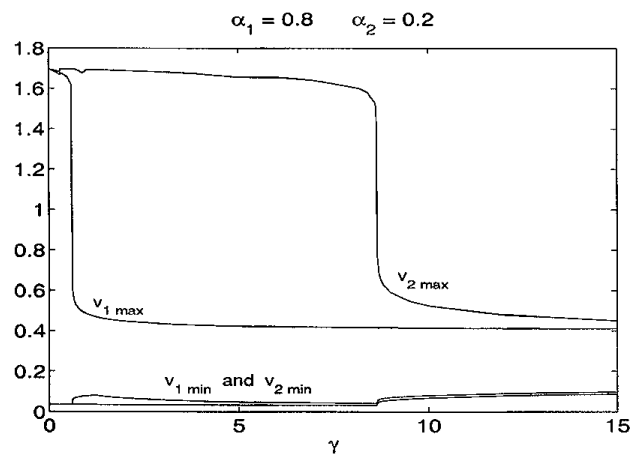

b)

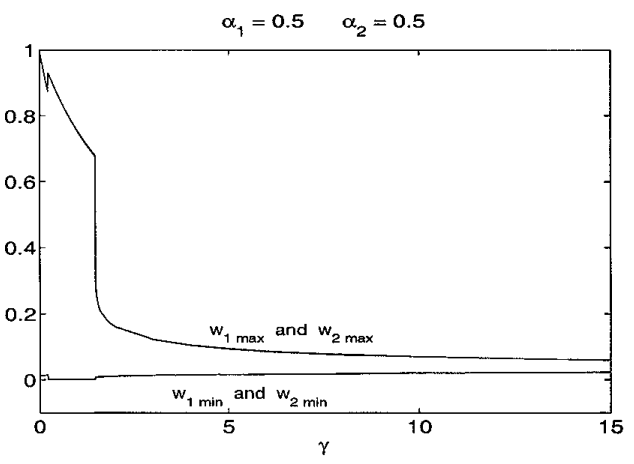

d)

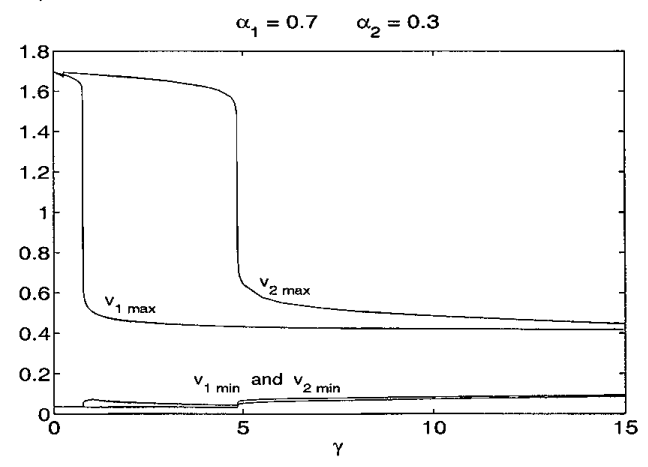

f)

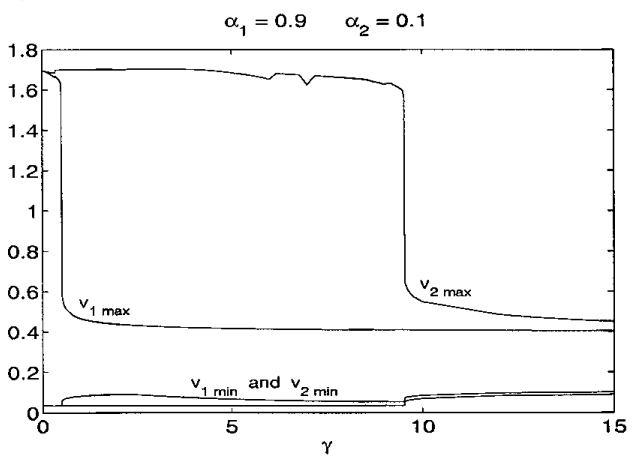

FIG. 7. Amplitude of the limit cycle for 2 globally coupled $(M=2)$ MFHN oscillators as a function of the global feedback parameter $\gamma$ and for different values of the fraction of oscillators in each cluster. The parameters are as in Figure 2 and (a) $\alpha_{1}=0.5$, v-amplitude, (b) $\alpha_{1}=0.5$, w-amplitude, (c) $\alpha_{1}=0.6$, v-amplitude, (d) $\alpha_{1}=0.7$, v-amplitude, (e) $\alpha_{1}=0.8$, v-amplitude, (f) $\alpha_{1}=0.9$, v-amplitude.

of $\alpha_{k}, k=1,2$. The amplitude of the oscillatory solutions for $v_{k}$ is represented as the minimum and maximum values $v_{k, \min }$ and $v_{k, \max }$, respectively. In Table 1 we present numerical approximations of $\gamma_{1, c}$ and $\gamma_{2, c}$ for different values of $\alpha_{1}$ and $\alpha_{2}$. For $\alpha_{1}=0.6,0.7$, and 0.8 we found that the localized solutions corresponding to a subset of values of $\gamma$ included in $\left(\gamma_{1, c}, \gamma_{2, c}\right)$ are 100-stable. 
TABLE 1

Localized solution for the MFHN model. Values of the canard critical values $\gamma_{c, 1}$ and $\gamma_{c, 2}$ as a function of the fraction of oscillators in each cluster. The values of the parameters are as in Figure 2 with $M=2$. The intervals of 100-stability, $I$, are (i) $I=1.01$ for $\alpha_{1}=0.6$, (ii) $I=0.6$ for $\alpha_{1}=0.7$ and $I=0.05$ for $\alpha_{1}=0.8$. For $\alpha_{1}=0.9$ 100-stable localized solutions were not found.

\begin{tabular}{|l|l|l|l|}
\hline$\alpha_{1}$ & $\alpha_{2}$ & $\gamma_{c, 1}$ & $\gamma_{c, 2}$ \\
\hline \hline 0.5 & 0.5 & 1.47 & 1.47 \\
\hline 0.6 & 0.4 & 1.01 & 2.40 \\
\hline 0.7 & 0.3 & 0.77 & 4.85 \\
\hline 0.8 & 0.2 & 0.6 & 8.64 \\
\hline 0.9 & 0.1 & 0.5 & 9.53 \\
\hline
\end{tabular}

The analysis presented in this section can be generalized for larger values of $M$, in which case we will have two regimes (LAO and SAO), but in each of the regimes we can have different amplitudes or phases for different clusters.

7. Discussion. In this paper we analyze the mechanism of localization of oscillations for a globally coupled system of relaxation oscillators of FHN type. In addition to localization, these models display the basic features of the modified Oregonator models studied in [6] and [5] to reproduce the experimental results: shape of the nullclines, a limit cycle created in a supercritical Hopf bifurcation, and display of canards among others.

Although the present study is motivated by the BZ reaction, a spatially extended system, experimental evidence suggests that the phenomena studied here, the mechanism of localization or creation of localized clusters, does not depend on diffusion $[4,28]$. Based on the results of the simulations presented in $[6]$ and simulations performed by the authors and not presented here, we conjecture that the diffusion plays an important role in spatially grouping together oscillators belonging to the same cluster.

We analyzed the canard phenomenon induced by the global feedback parameter $\gamma$ for bulk oscillations $(M=1)$, obtaining an expression for the canard critical value $\lambda_{c}$ and the critical global feedback parameter $\gamma_{c}$ as functions of the parameters of the models considered. We showed that, by increasing the value of the global feedback parameter, the canard phenomenon is induced for a critical value $\gamma_{c}$; i.e., as $\gamma$ passes $\gamma_{c}$ the system rapidly changes from an LAO regime to an SAO regime due to selfinhibition. Our numerical stability calculations show that this limit cycle need not be 100-stable in a neighborhood of $\gamma_{c}$; e.g., for values of $\gamma$ close enough to $\gamma_{c}$, bulk oscillations lose stability, generating other patterns, among them localized structures. The idea of induction of the canard phenomenon by changing $\gamma$ is a key to the analysis of localization.

We used the idea of self-inhibition to partially explain the two-cluster localization phenomenon $(M=2)$ for a system of FHN-type equations. We applied the reduction of dimension via clusters, and we analyzed each of the two oscillators separately, considering each as a forcing exerted on the other. By writing the equations for the nullclines of each oscillator, we saw that their dynamics can be understood as a combination of two phenomena: self-inhibition of each oscillator and inhibition (forcing) exerted on each oscillator by the remaining ones. Self-inhibition creates intervals of critical values of $\lambda$ and $\gamma$ given by (31) and (32), respectively. The forcing exerted on each oscillator by the other one changes the values of $\lambda_{k}(t)$. We did not analyze the effect of the forcing exerted on each oscillator by the remaining one, but 
we studied this effect numerically, showing that the main features of localization are present; i.e., the larger cluster is in an SAO regime, and the larger the size difference between two clusters the larger the interval of values of $\gamma$ for which the system has a localized solution, which is 100-stable. Our analysis reveals that for the VDP equations, localization is produced by self-inhibition alone. For systems that are not of FHN type (e.g., the BZ equations [6]), the analysis becomes more complicated.

In experiments on the BZ reaction with global inhibitory feedback $[4,5]$ as well as in simulations using an Oregonator model [5] and another BZ model [6], localized structures consisted of three clusters, the largest cluster in an SAO regime and two smaller phase-locked clusters in an LAO regime. The mechanism we propose here for FHN-type models does not deal with the multiple clusters in LAO regimes but does explain the counterintuitive inverse relation between amplitude regime and cluster size and sheds light on the role of self-inhibition in the phenomenon of localization.

We conjecture that a similar mechanism is responsible for localization in a modified Oregonator model for the BZ reaction [6] that we study in a forthcoming paper, as well as in the Oregonator model [5]. The canard phenomenon for a single twodimensional Oregonator model has been studied in [29], although in this case the Hopf bifurcation taking place in a neighborhood of the minimum of the activator nullcline may be subcritical instead of supercritical; then SAO are not possible for a single oscillator, though they might be possible in a globally coupled system. Our preliminary analysis shows that global feedback changes the stability type of the Hopf bifurcation point, thus allowing for SAO.

Appendix A. Calculation of the canonical form. The first step in calculating the canard critical value for system (13) is to transform it into its canonical form. We assume $\left(v_{m}, w_{m}\right)=0$.

We first expand the right-hand sides in both equations in (13) in Taylor series:

$$
\left\{\begin{array}{l}
F(v, w)=-b w+a v^{2}+H_{1}(v, w) \\
G(v, w, \lambda)=e v-c \lambda+d w+H_{2}(v, w, \lambda)
\end{array}\right.
$$

where

$$
\begin{gathered}
a=\frac{1}{2} \frac{\partial^{2} F}{\partial v^{2}}(\mathbf{0}), \quad b=\left|\frac{\partial F}{\partial w}(\mathbf{0})\right|, \\
c=\left|\frac{\partial G}{\partial \lambda}(\mathbf{0})\right|, \quad d=\frac{\partial G}{\partial w}(\mathbf{0}), \quad e=\frac{\partial G}{\partial v}(\mathbf{0}), \\
H_{1}(v, w)=\frac{\partial^{2} F}{\partial v w}(\mathbf{0}) v w+\frac{1}{6} \frac{\partial^{3} F}{\partial v^{3}}(\mathbf{0}) v^{3}+\mathcal{O}\left(w^{2}, v^{2} w, v w^{2}, w^{3}\right), \\
H_{2}(v, w, \lambda)=\frac{1}{2} \frac{\partial^{2} G}{\partial v^{2}}(\mathbf{0}) v^{2}+\frac{\partial^{2} G}{\partial v \lambda}(\mathbf{0}) v \lambda+\mathcal{O}\left(w^{2}, \lambda^{2}, v w, w \lambda\right) .
\end{gathered}
$$

In (34) and (36) $\mathbf{0}=(0,0)$, whereas in (35) and (37) $\mathbf{0}=(0,0,0)$.

Next, we substitute (33) into (13), getting

$$
\left\{\begin{array}{l}
v^{\prime}=-b w+a v^{2}+H_{1}(v, w) \\
w^{\prime}=\epsilon\left[e v-c \lambda+d w+H_{2}(v, w, \lambda)\right] .
\end{array}\right.
$$


Finally, we rescale system (38) by defining

$$
\begin{gathered}
V=e^{1 / 2} b^{1 / 2} a^{-1}, \quad W=e a^{-1}, \quad L=e^{3 / 2} b^{1 / 2} a^{-1} c^{-1}, \quad T=e^{-1 / 2} b^{-1 / 2}, \\
\hat{v}=\frac{v}{V}, \quad \hat{w}=\frac{w}{W}, \quad \hat{\lambda}=\frac{\lambda}{L}, \quad \hat{t}=\frac{t}{T}, \\
\hat{H}_{1}(\hat{v}, \hat{w})=\frac{T}{V} H_{1}(V \hat{v}, W \hat{w}) \\
=T W \frac{\partial^{2} F}{\partial \hat{v} \hat{w}}(\mathbf{0}) \hat{v} \hat{w}+\frac{1}{6} T V^{2} \frac{\partial^{3} F}{\partial \hat{v}^{3}}(\mathbf{0}) \hat{v}^{3}+\mathcal{O}\left(\hat{w}^{2}, \hat{v}^{2} \hat{w}, \hat{v} \hat{w}^{2}, \hat{w}^{3}\right) \\
=\hat{w}\left[T W \frac{\partial^{2} F}{\partial \hat{v} \hat{w}}(\mathbf{0}) \hat{v}+\mathcal{O}(\hat{w})\right]+\hat{v}^{2}\left[\frac{1}{6} T V^{2} \frac{\partial^{3} F}{\partial \hat{v}^{3}}(\mathbf{0}) \hat{v}+\mathcal{O}(\hat{w})\right],
\end{gathered}
$$

$$
\begin{gathered}
\hat{H}_{2}(\hat{v}, \hat{w}, \hat{\lambda})=\frac{T}{W} H_{2}(V \hat{v}, W \hat{w}, L \hat{\lambda}) \\
=\frac{T V^{2}}{2 W} \frac{\partial^{2} G}{\partial \hat{v}^{2}}(\mathbf{0}) \hat{v}^{2}+\frac{T V L}{W} \frac{\partial^{2} G}{\partial \hat{v} \hat{\lambda}}(\mathbf{0}) \hat{v} \hat{\lambda}+\mathcal{O}\left(\hat{w}^{2}, \hat{\lambda}^{2}, \hat{v} \hat{w}, \hat{w} \hat{\lambda}\right) \\
=\hat{v}\left[\frac{T V^{2}}{2 W} \frac{\partial^{2} G}{\partial \hat{v}^{2}}(\mathbf{0}) \hat{v}+\mathcal{O}(\hat{w})\right]+\hat{\lambda}\left[\frac{T V L}{W} \frac{\partial^{2} G}{\partial \hat{v} \hat{\lambda}}(\mathbf{0}) \hat{v}+\mathcal{O}(\hat{w}, \hat{\lambda})\right]
\end{gathered}
$$

and substituting (39)-(42) into (38). Calling

$$
h_{1}(\hat{v}, \hat{w})=-T W \frac{\partial^{2} F}{\partial \hat{v} \hat{w}}(\mathbf{0}) \hat{v}+\mathcal{O}(\hat{w})=b^{-1 / 2} e^{1 / 2} a^{-1} \frac{\partial^{2} F}{\partial \hat{v} \hat{w}}(\mathbf{0}) \hat{v}+\mathcal{O}(\hat{w}),
$$

$$
h_{2}(\hat{v}, \hat{w})=\frac{1}{6} T V^{2} \frac{\partial^{3} F}{\partial \hat{v}^{3}}(\mathbf{0}) \hat{v}+\mathcal{O}(\hat{w})=\frac{1}{6} e^{1 / 2} b^{1 / 2} a^{-2} \frac{\partial^{3} F}{\partial \hat{v}^{3}}(\mathbf{0}) \hat{v}+\mathcal{O}(\hat{w})
$$

$$
\begin{gathered}
h_{3}(\hat{v}, \hat{w}, \hat{\lambda})=\frac{T V^{2}}{2 W} \frac{\partial^{2} G}{\partial \hat{v}^{2}}(\mathbf{0}) \hat{v}+\mathcal{O}(\hat{w})=\frac{1}{2} e^{-1 / 2} b^{1 / 2} a^{-1} \frac{\partial^{2} G}{\partial \hat{v}^{2}}(\mathbf{0}) \hat{v}+\mathcal{O}(\hat{w}), \\
h_{4}(\hat{v}, \hat{w}, \hat{\lambda})=-\frac{T V L}{W} \frac{\partial^{2} G}{\partial \hat{v} \hat{\lambda}}(\mathbf{0}) \hat{v}+\mathcal{O}(\hat{w}, \hat{\lambda}) \\
=-e^{1 / 2} a^{-1} b^{1 / 2} c^{-1} \frac{\partial^{2} G}{\partial \hat{v} \hat{\lambda}}(\mathbf{0}) \hat{v}+\mathcal{O}(\hat{w}, \hat{\lambda}), \\
h_{5}(\hat{v}, \hat{w}, \hat{\lambda})=d T+\mathcal{O}(\hat{v}, \hat{w}, \hat{\lambda})=d e^{-1 / 2} b^{-1 / 2}+\mathcal{O}(\hat{v}, \hat{w}, \hat{\lambda}),
\end{gathered}
$$


rearranging terms we get the canonical form

$$
\left\{\begin{array}{l}
\hat{v}^{\prime}=-\hat{w}+\hat{v}^{2}-\hat{w} h_{1}(\hat{v}, \hat{w})+\hat{v}^{2} h_{2}(\hat{v}, \hat{w}), \\
\hat{w}^{\prime}=\epsilon\left[\hat{v}-\hat{\lambda}+\hat{v} h_{3}(\hat{v}, \hat{w}, \hat{\lambda})-\hat{\lambda} h_{4}(\hat{v}, \hat{w}, \hat{\lambda})+\hat{w} h_{5}(\hat{v}, \hat{w}, \hat{\lambda})\right] .
\end{array}\right.
$$

Note that in (48) the sign ' represents $d / d \hat{t}$.

Appendix B. Calculation of the canard critical value. In [16] an expression for $\hat{\lambda_{c}}$ was found:

$$
\hat{\lambda_{c}}=\frac{-a_{1}+3 a_{2}-2 a_{3}+2 a_{5}}{8} \epsilon+\mathcal{O}\left(\epsilon^{3 / 2}\right),
$$

where

$$
a_{1}=\frac{\partial h_{1}}{\partial \hat{v}}, \quad a_{2}=\frac{\partial h_{2}}{\partial \hat{v}}, \quad a_{3}=\frac{\partial h_{4}}{\partial \hat{v}}, \quad a_{5}=h_{5} .
$$

Substituting (34), (35), and (43)-(47) into (50), we get

$$
\begin{array}{cc}
a_{1}=-\frac{2 G_{v}^{1 / 2} F_{v w}}{\left|F_{w}\right|^{1 / 2} F_{v v}}, & a_{2}=\frac{2 G_{v}^{1 / 2}\left|F_{w}\right|^{1 / 2} F_{v v v}}{3 F_{v v}^{2}}, \\
a_{3}=\frac{\left|F_{w}\right|^{1 / 2} G_{v v}}{G_{v}^{1 / 2} F_{v v}}, & a_{5}=\frac{G_{w}}{G_{v}^{1 / 2}\left|F_{w}\right|^{1 / 2}},
\end{array}
$$

where all the functions are calculated at $\mathbf{0}$. The corresponding expression for $\lambda_{c}=L \hat{\lambda_{c}}$ is

$$
\begin{gathered}
\lambda_{c}(\sqrt{\epsilon})=-\frac{g_{v}^{3 / 2}\left|F_{w}\right|^{1 / 2}}{4 F_{v v}\left|G_{\lambda}\right|}\left[-a_{1}+3 a_{2}-2 a_{3}+2 a_{5}\right] \epsilon+\mathcal{O}\left(\epsilon^{3 / 2}\right) \\
=-\frac{G_{v}}{2 F_{v v}^{3}\left|G_{\lambda}\right|}\left[G_{v} F_{v w} F_{v v}+G_{v}\left|F_{w}\right| F_{v v v}\right. \\
\left.\quad-\left|F_{w}\right| G_{v v} F_{v v}+G_{w} F_{v v}^{2}\right] \epsilon+\mathcal{O}\left(\epsilon^{3 / 2}\right),
\end{gathered}
$$

where all the functions are calculated at $\mathbf{0 .}$.

If $F$ were not independent of $\epsilon$, then we would need to add a term $\epsilon h_{6}$ in (48). This would produce an additional $\mathcal{O}(\epsilon)$ term, proportional to $d h_{6} / d v$, in the expression for $\lambda_{c}[15]$.

Appendix C. Equilibrium point and Hopf bifurcation. Here we present a result by Krupa and Szmolyan [15]. Based on the calculations from appendices A and B, we apply it to system (1) and the examples presented in section 2.

Consider system (1) with $\gamma=0$ and $\left(v_{k}, w_{k}\right)$ replaced by $(v, w)$. Call

$$
A=-a_{1}+3 a_{2}-2 a_{3}-2 a_{5} .
$$

Assume the following:

(i) The critical manifold $\{(v, w): F(v, w)=0\}$ can be written in the form $w=$ $f(v)$, and the function $f$ is cubic-like, i.e., it has precisely two critical points, one nondegenerate minimum and one nondegenerate maximum, each of which satisfies $\partial^{2} F / \partial v^{2}(\mathbf{p}) \neq \mathbf{0}$ and $\partial F / \partial w(\mathbf{p}) \neq \mathbf{0}$. Without loss of generality, the minimum of $f$ can be taken as $(0,0)$. 
(ii) For $\epsilon=0$ the left and right branches of the critical manifold $F(v, w)=0$ are attracting and the central branch is repelling.

(iii) For $\lambda=0$ the fold point $(0,0)$ is a nondegenerate canard point; i.e., it satisfies $\partial G / \partial v(\mathbf{0}) \neq \mathbf{0}$ and $\partial G / \partial \lambda(\mathbf{0}) \neq \mathbf{0}$.

(iv) When $\lambda=0, v^{\prime}<0$ for the slow flow on the right branch of $f$ and $v^{\prime}>0$ for the slow flow on the central and left branches of $f$, including the point $(0,0)$.

Then there exist $\epsilon_{0}>0$ and $\lambda_{0}>0$ such that, for each $0<\epsilon<\epsilon_{0},|\lambda|<\lambda_{0}$, system (1) with $\gamma=0$ and $\left(v_{k}, w_{k}\right)$ replaced by $(v, w)$ has precisely one equilibrium point $p_{e}$ in a neighborhood of the origin which converges to the canard point as $(\epsilon, \lambda) \rightarrow(0,0)$. Moreover, there exists a curve

$$
\lambda_{H}(\sqrt{\epsilon})=-\frac{a_{5}}{2} \epsilon+\mathcal{O}\left(\epsilon^{3 / 2}\right)
$$

such that $p_{e}$ is stable (unstable) for $\lambda<\lambda_{H}\left(\lambda>\lambda_{H}\right.$ ). The equilibrium point $p_{e}$ loses stability through a supercritical (subcritical) Hopf bifurcation if $A>0(A<0)$.

The proof is given in [15].

By substituting (51) and (52) into (54) we get

$A=\frac{2}{\left|F_{w}\right|^{1 / 2}\left(G_{v}\right)^{1 / 2}\left(F_{v v}\right)^{2}}\left[G_{v} F_{v v} F_{v w}+\left|F_{w}\right| G_{v} F_{v v v}-\left|F_{w}\right| G_{v v} F_{v v}-G_{w}\left(F_{v v}\right)^{2}\right]$.

Note that for the FHN equations (examples (ii) and (iii) in section 2) the condition for a supercritical Hopf bifurcation is equivalent to $3 h g_{v}+a g_{v v}>2 a^{2}$. In particular, for the classical FHN equations (example (ii) in section 2) this becomes $3 h \beta>2 a^{2}$.

By substituting the second equation in (52) into (55) we get

$$
\lambda_{H}(\sqrt{\epsilon})=-\frac{G_{w}}{2\left(G_{v}\right)^{1 / 2}\left|F_{w}\right|^{1 / 2}} \epsilon+\mathcal{O}\left(\epsilon^{3 / 2}\right) .
$$

Note that for the FHN equations $\lambda_{H}(\sqrt{\epsilon})=1 /\left(G_{v}\right)^{1 / 2} \epsilon+\mathcal{O}\left(\epsilon^{3 / 2}\right)$, and for the classical FHN equations $\lambda_{H}(\sqrt{\epsilon})=1 / \beta^{1 / 2} \epsilon+\mathcal{O}\left(\epsilon^{3 / 2}\right)$.

Acknowledgments. The authors thank Tasso Kaper and Kresimir Josic for helpful comments on the mathematical treatment of the canard phenomenon, Milos Dolnik for useful comments on the BZ reaction, and the canard group at Boston University for the collective learning experience on the mathematics of canards.

\section{REFERENCES}

[1] J. D. Murray, Mathematical Biology, Springer-Verlag, Berlin, 1989.

[2] I. R. Epstein and K. Showalter, Nonlinear chemical dynamics: Oscillations, patterns and chaos, J. Phys. Chem., 100 (1996), pp. 13132-13147.

[3] S. H. Strogatz, Nonlinear Dynamics and Chaos, Addison-Wesley, Reading, MA, 1994.

[4] V. K. Vanag, L. Yang, M. Dolnik, A. M. Zhabotinsky, and I. R. Epstein, Oscillatory cluster patterns in a homogeneous chemical system with global feedback, Nature, 406 (2000), pp. 389-391.

[5] V. K. Vanag, A. M. Zhabotinsky, and I. R. Epstein, Pattern formation in the BelousovZhabotinsky reaction with photochemical global feedback, J. Phys. Chem., 104A (2000), pp. 11566-11577.

[6] L. Yang, M. Dolnik, A. M. Zhabotinsky, and I. R. Epstein, Oscillatory clusters in a model of the photosensitive Belousov-Zhabotinsky reaction system with global feedback, Phys. Rev. E, 62 (2000), pp. 6414-6420. 
[7] A. M. Zhabotinsky, F. Buchholtz, A. B. Kiyatkin, and I. R. Epstein, Oscillations and waves in metal-ion-catalyzed bromate oscillating reactions in highly oxidized states, J. Phys. Chem., 97 (1993), pp. 7578-7584.

[8] R. Kuske And T. ERneux, Localized synchronization of two coupled solid state lasers, Optics Communications, 139 (1997), pp. 125-131.

[9] R. Kuske And T. ERneux, Bifurcation to localized oscillations, European J. Appl. Math., 8 (1997), pp. 389-402.

[10] M. Boukalouch, J. Elezgaray, A. Arneodo, J. Boissonade, and P. De Kepper, Oscillatory instability induced by mass interchange between two coupled steady-state reactors, J. Phys. Chem., 91 (1987), pp. 5843-5845.

[11] F. Dumortier and R. Roussarie, Canard Cycles and Center Manifolds, Mem. Amer. Math. Soc., 121 (1996).

[12] W. Eckhaus, Relaxation oscillations including a standard chase on French ducks, in Asymptotic Analysis, II, Lecture Notes in Math. 985, Springer-Verlag, Berlin, 1983, pp. 449-497.

[13] S. M. BAER AND T. ERneux, Singular Hopf bifurcation to relaxation oscillations, SIAM J. Appl. Math., 46 (1986), pp. 721-739.

[14] E. Benoit, J. L. Callot, F. Dienner, and M. Dienner, Chasse au Canard, IRMA, Strasbourg, France, 1980.

[15] M. Krupa and P. Szmolyan, Relaxation oscillation and canard explosion, J. Differential Equations, 174 (2001), pp. 312-368.

[16] M. KRUPA AND P. SzMOlyan, Extending geometric singular perturbation theory to nonhyperbolic points - fold and canard points in two dimensions, SIAM J. Math. Anal., 33 (2001), pp. $286-314$.

[17] M. Brons And K. BAR-Eli, Canard explosion and excitation in a model of the BZ reaction, J. Phys. Chem., 95 (1991), pp. 8706-8713.

[18] F. Buchholtz, M. Dolnik, and I. R. Epstein, Diffusion-induced instabilities near a canard, J. Phys. Chem., 99 (1995), pp. 15093-15101.

[19] H. G. Rotstein, N. Kopell, A. Zhabotinsky, and I. R. Epstein, Canard phenomenon and localization of oscillations in the Belousov-Zhabotinsky reaction with global feedback, submitted.

[20] H. G. Rotstein And R. Kuske, personal communication.

[21] H. G. Rotstein, R. Kuske, and N. Kopell, Localized Oscillations in a Coupled Two-Pool Model. A Canard Mechanism, in preparation.

[22] R. L. Burden and J. D. Faires, Numerical Analysis, PWS Publishing, Boston, 1980.

[23] R. FitzHugh, Impulses and physiological states in models of nerve membrane, Biophys. J., 1 (1961), pp. 445-466.

[24] F. C. Hoppensteadt, An Introduction to the Mathematics of Neurons, Cambridge University Press, New York, 1996.

[25] E. F. Mishchenko And N. Kh. Rozov, Differential Equations with Small Parameters and Relaxation Oscillations, Plenum Press, New York, London, 1980.

[26] J. Grasman, Asymptotic Methods for Relaxation Oscillations and Applications, SpringerVerlag, New York, 1986.

[27] F. DumoRTier, Techniques in the theory of local bifurcations: Blow-up, normal forms, nilpotent bifurcations, singular perturbations, in Bifurcations and Periodic Orbits of Vector Fields, D. Schlomiuk, ed., Kluwer Academic Press, Dordrecht, The Netherlands, 1993, pp. 19-73.

[28] V. K. Vanag, A. M. Zhabotinsky, and I. R. Epstein, Oscillatory clusters in the periodically illuminated, spatially extended Belousov-Zhabotinsky reaction, Phys. Rev. Lett., 86 (2001), pp. $552-555$.

[29] M. Krupa, W. F. Langford, and J. P. Voroney, Canard Explosion in the Oregonator, in preparation. 